\title{
Identification of a non-coding KLF4 transcript generated from intron retention and downregulated in human hepatocellular carcinoma
}

\author{
JUNWEI ZHOU ${ }^{1}$, PAUL BO-SAN LAI ${ }^{2}$ and STEPHEN KWOK-WING TSUI ${ }^{1}$ \\ ${ }^{1}$ School of Biomedical Sciences, ${ }^{2}$ Department of Surgery, The Chinese University of Hong Kong, \\ Shatin, N.T., Hong Kong SAR, P.R. China
}

Received May 20, 2015; Accepted June 29, 2015

DOI: $10.3892 /$ ijo.2015.3104

\begin{abstract}
The Krüppel-like factor 4 (KLF4) gene is related to various biological processes including stem cell reprogramming and tumorigenesis. In this study, we identified and characterized a non-coding transcript of KLF4, which was designated KLF4-003, in human liver tissue samples. KLF4-003 was identified in a number of cell lines by reverse transcription PCR and DNA sequencing. Its expression levels were determined in 54 pairs of human hepatocellular carcinoma (HCC) tissues and a number of HCC cell lines by real-time PCR (RT-PCR). Methylation status of KLF4-003 CpG islands was determined by bisulfite sequencing. The regulatory effect of KLF4-003 CpG islands hypermethylation in Hep3B cells was then validated by the 5-aza-dC demethylation treatment, followed by RT-PCR analysis. Receiver operating characteristic (ROC) curve was created to evaluate the diagnostic value for differentiating between HCC cancer and benign diseases. The association study between KLF4-003 expression level and clinical traits of HCC patients was performed with SPSS. We found that KLF4-003 was downregulated in 46 out of 54 HCC samples compared with their adjunct normal tissues. The reduced KLF4-003 expression was significantly associated with $\mathrm{HCC}$ recurrence $(\mathrm{P}=0.045)$ in the follow-up of 31 HCC patients. Significant differences were detected between the methylation status of $\mathrm{HCC}$ specimens and their adjacent
\end{abstract}

Correspondence to: Dr Stephen Kwok-Wing Tsui, School of Biomedical Sciences, The Chinese University of Hong Kong, Shatin, N.T., 39 Area, Hong Kong SAR, P.R. China

E-mail:kwtsui@cuhk.edu.hk

Abbreviations: KLF4, Krüppel-like factor 4; HCC, human hepatocellular carcinoma; RT-PCR, real-time polymerase chain reaction; ROC, receiver operating characteristic; AUC, area under ROC curve; ncRNAs, non-coding RNAs; lncRNAs, long non-coding RNAs; DMEM, Dulbecco's modified Eagle's medium; ORF, open reading frame; AJCC, American Joint Committee on Cancer

Key words: KLF4-003, hepatocellular carcinoma, CpG island, hypermethylation, long non-coding RNA normal controls. Demethylation treatment significantly rescued the expression of KLF4-003 in Hep3B cells. Such observation indicated that the $\mathrm{CpG}$ island hypermethylation was at least partially responsible for the downregulation of KLF4-003 in HCC. The area under ROC curve for the prediction of HCC reached 0.803 (95\% CI=0.719-0.886, $\mathrm{P}<0.001)$. Our results suggested that the expression of KLF4-003 was epigenetically regulated by methylation status of a KLF4-003 CpG island in HCC. The differential expression of KLF4-003 might play an important role in $\mathrm{HCC}$ development and might serve as a potential biomarker for the diagnosis of HCC.

\section{Introduction}

Hepatocellular carcinoma (HCC) is one of the most common malignant cancers and the third most fatal form of neoplasia worldwide. It is mostly associated with multiple factors including HBV/HCV virus infection $(1,2)$, exposure to hepatocarcinogens such as aflatoxin B1, and exposure to alcohol $(3,4)$. Previous studies have demonstrated multiple regulatory pathways involved in HCC. However, the exact mechanism for the pathogenesis of $\mathrm{HCC}$ is not yet clearly understood.

KLF4 gene is located on chromosome 9 and has 5 exons. Multiple transcripts of KLF4 and their protein isoforms (for example, KLF4 $\alpha$, KLF4 $\beta$, KLF4 $\gamma$ and KLF4 $\delta$ ) have been reported (5). However, little attention has been paid to the putative non-coding transcript variant, KLF4-003 (Ensembl: ENST00000493306) possibly due to the fact that it has no protein product.

Recent genomic and transcriptomic projects have unraveled an astounding large number of non-coding RNAs (ncRNAs) in the human genome $(6,7)$. A class of small ncRNAs has been identified, some of which have been linked to neoplastic transformation (8-11). Another class of ncRNAs, long ncRNAs (lncRNAs) were tentatively defined as ncRNAs with $>200$ nucleotides in length and featured with diversity of their sequences and complexity of the mechanisms involved. Accumulating evidence supports the possibility that lncRNA acts as genetic regulators or riboregulators (12). Altered lncRNA levels were found to be responsible for the aberrant expression of gene products that might be related to cancer biology. For example, metastasis associated lung 
adenocarcinoma transcript 1 , a human lncRNA was found upregulated in metastasizing non-small cell lung carcinomas and is evolutionarily conserved among mammals (13). Another lncRNA, the paternally imprinted gene H19 (14), can function as a tumor suppressor in some tumor types and may play a significant role in tumorigenesis in other types (15-17). It is notable that Hepcarcin, also an lncRNA transcript, was found highly upregulated in human HCCs as well as other five non-hepatocellular human carcinomas and may serve as an informative marker for HCCs. Although a few aberrantly expressed protein-coding genes and several lncRNAs have been identified in HCC, novel molecular markers with earlydiagnostic or risk-assessment value are still urgently needed. It is of paramount importance to elucidate the relationships between clinical traits and molecular changes in HCC for developing new strategies that can be used in HCC diagnosis, treatment and prognosis.

Extensive studies have been carried out on KLF4 in the field of stem cells and oncology, including a recent study showing that high cytoplasmic expression of KLF4 was associated with better disease-specific survival and was an independently favorable prognostic factor in HCC (18). However, little is known with regard to its non-coding transcript variants. Here, we report the identification of the splice variant KLF4-003 and elucidate the characteristics of this variant, including its differential expression and epigenetic regulation in $\mathrm{HCC}$, association with HCC traits and the potential diagnostic significance as a biomarker for HCC.

\section{Materials and methods}

Cell culture. All cell lines used in this study were purchased from the American Type Culture Collection. WRL68 (a human fetal liver cell line) and two human hepatocellular carcinoma cell lines including HepG2 and Huh7 were cultured in Dulbecco's modified Eagle's medium (DMEM) (Thermo Fisher Scientific, Inc., Waltham, MA USA) supplemented with $10 \%$ (v/v) fetal bovine serum, $100 \mathrm{U} / \mathrm{ml}$ penicillin and $100 \mu \mathrm{g} / \mathrm{ml}$ streptomycin, while Hep3B (a human hepatocellular carcinoma cell line) was cultured in Roswell Park Memorial Institute-1640 medium (Thermo Fisher Scientific, Inc.) with the same supplements. The other cells from different tissues including $\mathrm{HeLa}$ (from cervical cancer), HONE1 (from nasopharyngeal cancer), OB (from osteoblast), Saos2 (from osteosarcoma) and BMSC (bone marrow stromal cells) were also cultured in DMEM medium at $37^{\circ} \mathrm{C}, 5 \% \mathrm{CO}_{2}$ humidified incubator.

Patient samples. Cancerous HCC tissues and adjacent nontumor tissue ( $\geq 1 \mathrm{~cm}$ away from the tumor edge) were collected from patients who underwent surgical resection at the Prince of Wales Hospital, Hong Kong, China. Written consents was obtained from each patient prior to tissue harvesting and the study protocol was approved by the ethics committee of the Chinese University of Hong Kong. The tissues were immediately snap-frozen in liquid nitrogen and stored at $-80^{\circ} \mathrm{C}$ pending analysis. Further details of the patients are given in Table I.

RNA extraction, reverse transcription PCR, DNA sequencing for KLF4-003. Total RNAs from different cell lines or HCC tissues were extracted by using TRIzol reagent (Thermo Fisher Scientific, Inc.) following the manufacturer's instructions. Nuclear and cytoplasmic RNAs were extracted by using the NE-PER Nuclear and Cytoplasmic Extraction reagents (Thermo Fisher Scientific, Inc.) followed by TRIzol reagent extraction. cDNA was synthesized by $1 \mu \mathrm{g}$ RNA with QuantiTect Rev Transcription kit (Qiagen, GmbH. Hilden, Germany) according to the protocol provided by the manufacturer. The reverse transcription reaction mixture was incubated at $42^{\circ} \mathrm{C}$ for $15 \mathrm{~min}$ for reverse transcription followed by denaturation at $95^{\circ} \mathrm{C}$ for $3 \mathrm{~min}$. KLF4-003 in each cell line was identified with PCR amplification followed by DNA sequencing (service provided by $\mathrm{BGI}$ ). The PCR reaction contains $1.25 \mathrm{U}$ of AmpliTaq Gold DNA polymerase (Applied Biosystems, Foster City, CA, USA), 1X PCR buffer, $200 \mu \mathrm{M}$ dNTP mixture, and $200 \mathrm{nM}$ of each primer in a final volume of $50 \mu \mathrm{l}$. The forward primer sequence is 5'-TCC CGG CTT CCA TCC CCA CCC-3'. The reverse primer is 5'-GGT CCT TTT CCG GGG CCA CGA TC-3'. PCR were performed by DNA denaturing at $94^{\circ} \mathrm{C}$ for $3 \mathrm{~min}$, followed by 35 cycles of $94^{\circ} \mathrm{C}$ for $30 \mathrm{sec}, 60^{\circ} \mathrm{C}$ for $30 \mathrm{sec}, 72^{\circ} \mathrm{C}$ for $1 \mathrm{~min}$ with a final extension of $72^{\circ} \mathrm{C}$ for $7 \mathrm{~min}$. For all primers, the same PCR condition was used.

Bioinformatics analysis. The exon-intron patterns of KLF4-003 transcript were constructed by Ensembl browser tool (http:// asia.ensembl.org/index.html). The $\mathrm{CpG}$ island was revealed with Genome Browser tool of UCSC (University of California, Santa Cruz) (http://genome.ucsc.edu/cgiBin/hgGateway).

Bisulfite sequencing. Genomic DNA was extracted from cells or frozen tissues using QIAamp DNA Mini kit (Qiagen, $\mathrm{GmbH}$ ) following the manufacturer's instructions. Sodium bisulphate treatment was performed with the EZ DNA Methylation-Gold kit (Zymo Research, Freiburg, Germany). Twenty nanograms of treated DNA were used as a template for PCR amplification using ZymoTaq DNA polymerase (Zymo Research) and primers targeting a fragment of non-promoter CpG island proximal to the retained intron of KLF4-003. Forward primer, 5'-GGT TTT TAG TTT ACG TTG TAT AGT GTT GG-3' and reverse primer, 5'-CCG TAA CGC CAA CCA AAC AAC T-3' were used for PCR amplification. The PCR products were cloned into pGEM-T Easy vector (Promega, Madison, WI, USA) with the manufacturer's standard protocol for sequencing to determine the methylation status of the CpG sites within KLF4-003 gene. In total, 73 colonies were sequenced.

5-aza-2'-deoxycytidine (5-aza-dC) treatment. Cells were seeded at a density of $1 \times 10^{5}$ cells per well in 6 -well plate one day before treatment. Dimethyl sulfoxide dissolved 5-aza-dC (Sigma, St. Louis, MO, USA), a demethylating agent, was added to the cells at different concentration, 0, 2 or $5 \mu \mathrm{M}$. The 5 -aza-dC and medium were refreshed every day. The cells were harvested for total RNA extraction 4 days posttreatment. The expression level of KLF4-003 was determined by real-time (RT) PCR.

Cloning of KLF4-003. To construct the pCMV-MycKLF4-003 vector, PCR was performed by gene specific 
Table I. Demographic and clinical features of HCC patients.

\begin{tabular}{lrccc}
\hline & & \multicolumn{2}{c}{ KLF4-003 expression } & \\
\cline { 3 - 4 } $\begin{array}{l}\text { Pathological } \\
\text { parameter }\end{array}$ & Total & Reduced & Non-reduced & P-value \\
\hline Gender & & & & \\
Male & 42 & 36 & 6 & 1.000 \\
Female & 12 & 10 & 2 & \\
Age & & & & \\
$<60$ & 34 & 29 & 5 & 1.000 \\
$\geq 60$ & 20 & 17 & 3 & \\
HBsAg & & & & \\
Positive & 45 & 39 & 6 & 0.607 \\
Negative & 9 & 7 & 2 & \\
AJCC staging & & & & \\
Stage I & 38 & 31 & 7 & 0.342 \\
Stage II & 6 & 5 & 1 & \\
Stage III & 10 & 10 & 0 & \\
Cirrhosis & & & & \\
Yes & 24 & 22 & 2 & 0.230 \\
No & 30 & 24 & 6 & \\
Recurrence & & & & \\
Yes & 14 & 4 & 10 & 0.045 \\
No & 17 & 11 & 6 & \\
\hline & & & & \\
\hline Only & & & & \\
\hline
\end{tabular}

aOnly relative expression level $<0.25$ were grouped in 'reduced'.

primers containing $B g l \mathrm{II}$ site and $\mathrm{XhoI}$ site, respectively. The forward primer sequence is $5^{\prime}$-GGC AGA TCT TGG CTG TCA GCG ACG CGC T-3'. The reversed primer is 5'-GGC CTC GAG TTA AAA ATG CCT CTT CAT GTG T-3'. PCR products were purified using Wizard ${ }^{\mathrm{TM}}$ SV Gel and PCR Clean-up system (Promega) following the manufacturer's instructions. Purified PCR products and pCMV-Myc vector (Clontech, Mountain View, CA, USA) were submitted for BglII and XhoI (New England Biolabs, Ipswich, MA, USA) double digestion. Digestion products were resolved in $1 \%$ agarose gel, followed by purification of the DNA products and ligation with DNA insert using T4 ligase (New England Biolabs). The entire mixture of ligation products were transformed into E. coli competent cells (DH5 $\alpha$ ) followed by recovery in $800 \mu 12 \%$ LB (Luria Bertani) (USB Corp., Cleveland, OH, USA) medium. $E$. coli clones carrying the desired inserts were selected by ampicillin resistance and further cultured for plasmid extraction using the Mini Plus ${ }^{\mathrm{TM}}$ plasmid DNA Extraction system (Viogene, Sunnyvale, CA, USA). The sequence of cloned KLF4-003 in pCMV-Myc vector was determined and confirmed by automated DNA sequencing (service provided by BGI).

Transient transfection of KLF4-003 in human liver cell line. Hep3B cells were seeded in 6-well plate at an initial density of $2 \times 10^{5}$ cells per well. When the cells were $70 \%$ confluent, plasmid, pCMV-Myc, pCMV-KLF4-003 were transfected into the cells using Lipofectamine ${ }^{\mathrm{TM}} 2000$ (Thermo Fisher Scientific, Inc.) according to the manufacturer's procedures, respectively. Cells were incubated at $37^{\circ} \mathrm{C}$ in $5 \% \mathrm{CO}_{2}$ for 24-72 $\mathrm{h}$ prior to RT-PCR and western blot assay.

RT-PCR. RT-PCR was performed using $2 \mathrm{X}$ Power SYBR Green PCR Master Mix (Thermo Fisher Scientific, Inc.) with ABI 7500 Fast RT-PCR system (Thermo Fisher Scientific, Inc.). The PCR cycling conditions included an initial denaturation of $95^{\circ} \mathrm{C}$ for 10 min followed by 40 cycles of $95^{\circ} \mathrm{C}$ for $15 \mathrm{sec}$ and $60^{\circ} \mathrm{C}$ for $1 \mathrm{~min}$. KLF4-003 was amplified by forward primer 5'-TCG GGA CAC ACG GGA TGA T-3' paired with reverse primer 5'-GCC CGC GTA ATC ACA AGT GT-3'. While forward primer 5'-CAT TAC CAA GAG CTC ATG CC-3' paired with reverse primer 5'-GCC CGC GTA ATC ACA AGT GT-3' were used for KLF4 amplification. The house-keeping gene $\beta$-actin was measured for normalization using forward primer 5'-GCC CCG CGA GCA CAG AGC-3' paired with reverse primer 5'-TGC CGG AGC CGT TGT CGA-3'. Comparative $\mathrm{C}_{\mathrm{T}}$ method $\left(2^{-\Delta \Delta C T}\right)$ was used to calculate the relative level of the KLF4 and KLF4-003 mRNA.

Western blot analysis. Total proteins were extracted from Hep3B cells with RIPA lysis and extraction buffer (Thermo Fisher Scientific, Inc.) following the manufacturer's protocol. The target proteins were separated on sodium dodecyl sulfate polyacrylamide gel electrophoresis and transferred onto PVDF membrane. Specific antibodies including anti-Myc and anti-KLF4 (Santa Cruz Biotechnology, USA) were used for protein detection. The signals of target proteins were visualized on X-ray film after treatment with Western Lightning Chemiluminescence Reagent Plus (Perkin-Elmer Life Science, MA, USA).

Statistical analysis. Statistical significance between groups was analyzed by Student's t-test. Correlation analysis was used to examine the association between the expression of KLF4-003 and clinical parameters of HCC patients. Receiver operating characteristic (ROC) curve was plotted by SPSS (version 16.0) to evaluate the diagnostic value for differentiating between HCC cancer and benign diseases. The other figures were created with GraphPad Prism5 (GraphPad, San Diego, CA, USA). P-value of $\leq 0.05$ was considered statistically significant.

\section{Results}

Sequence analysis and expression profile of KLF4-003. According to the database of Ensembl, KLF4-003 is a noncoding transcript of KLF4 gene (ENSG00000136826). Compared with the vulgate transcript of KLF4, an intron retention was observed in KLF4-003 (Fig. 1A). This retained intron is 102 nucleotides in length and located near a nonpromoter CpG island in KLF4-003 gene (Fig. 1B). By RT-PCR and DNA sequencing, KLF4-003 transcript was identified in a series of cell lines including WRL68, HONE1, OB, BMSC, HeLa and Soas 2 cells (Fig. 1C). 
A

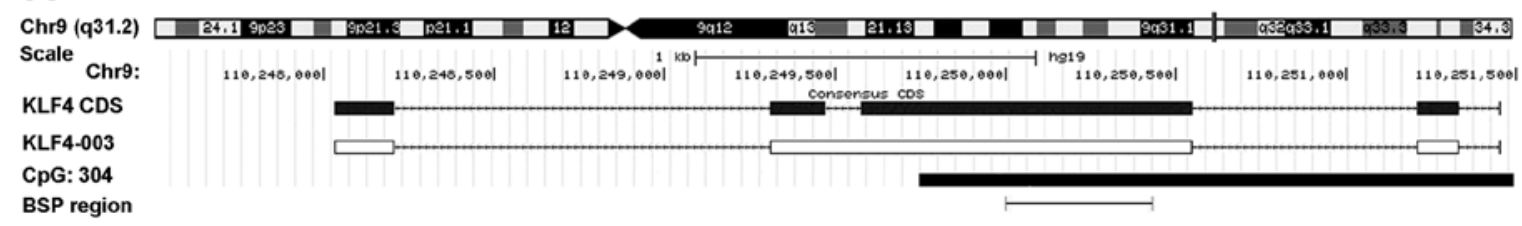

B

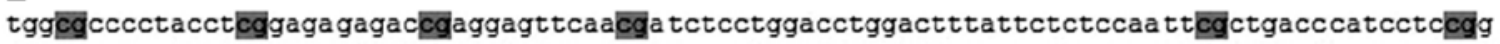

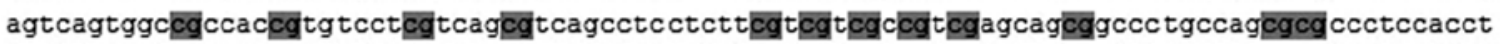

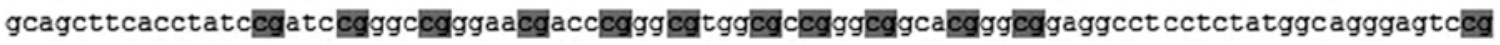

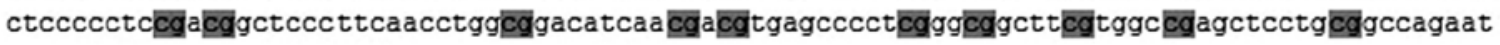

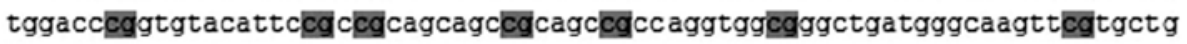

C

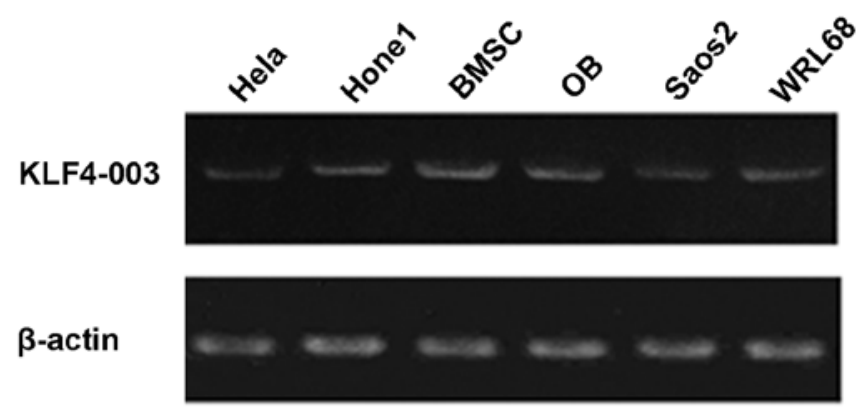

Figure 1. Chromosomal location, CpG island and expression profile of KLF4-003. (A) The chromosomal location of KLF4-003 with KLF4 CDS as reference. KLF4-003 is located on chromosome 9 (9q31.2). CpG island was revealed by UCSC Browser (http://genome.ucsc.edu/). The DNA region selected for bisulfite sequencing is indicated. (B) The target DNA sequence containing $47 \mathrm{CpG}$ sites in KLF4-003 CpG island for bisulfite sequencing. (C) Expression profile of KLF4-003 in various cell lines including HeLa, HONE1, BMSC, OB, Saos2 and WRL68 revealed by reverse transcription PCR with $\beta$-actin as a control.
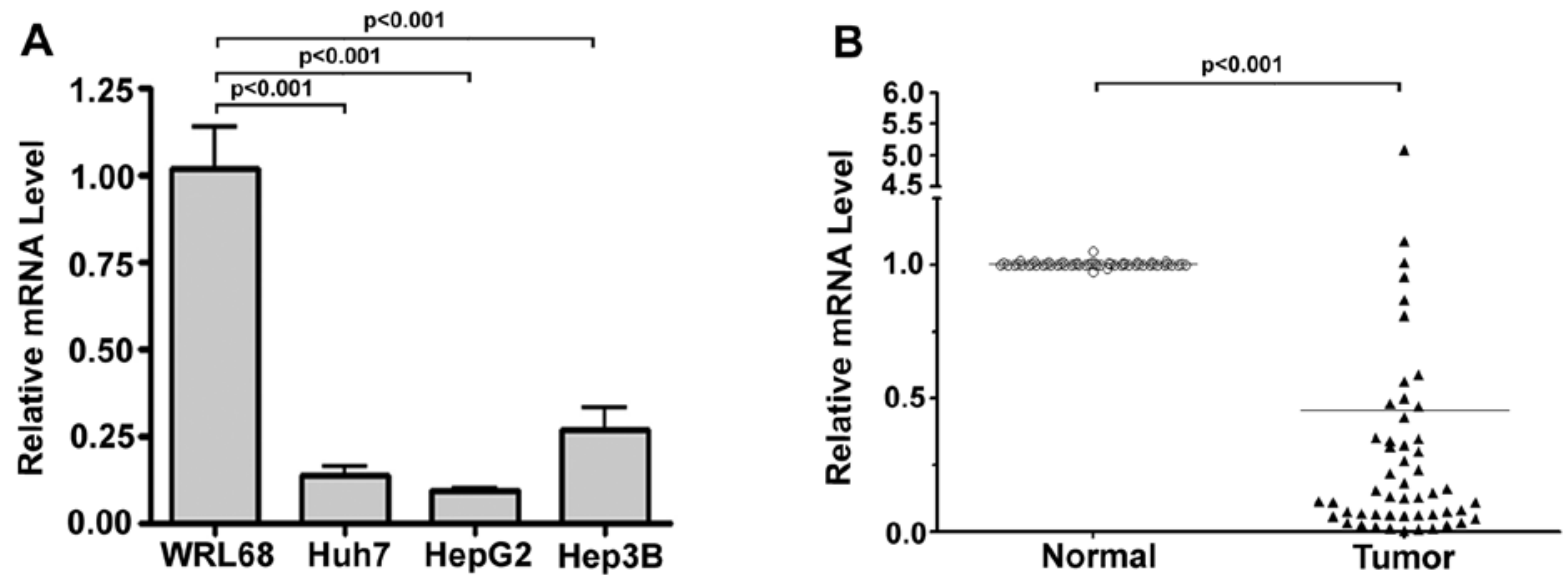

Figure 2. Downregulation of KLF4-003 in both HCC cell lines and HCC tissues. (A) The mRNA expression level of the KLF4-003 in four liver cell lines was determined by RT-PCR with $\beta$-actin gene as a housekeeping control. Compared with WRL68 normal liver cells, the expression of KLF4-003 mRNA was downregulated in HCC cell lines Huh7, Hep3B and HepG2. (B) The mRNA expression level of KLF4-003 in HCC patients were measured by RT-PCR. Significant downregulation of KLF4-003 was observed in 46 of 54 tumor samples.

KLF4-003 is downregulated in liver cancer cell lines and HCC tissues. To determine the expression levels of KLF4-003, RT-PCR was performed in a number of liver cancer cell lines using $\beta$-actin as a housekeeping control. Compared with WRL68 normal liver cells, the expression of KLF4-003 mRNA was significantly downregulated in the three examined HCC cell lines including Huh7, HepG2 and Hep3B ( $\mathrm{P}<0.001$, Fig. 2A).

We then examined the expression levels of KLF4-003 in the $\mathrm{HCC}$ tissues and their adjacent normal counterparts of HCC patients using RT-PCR. Among 54 pairs of clinical specimens,
KLF4-003 were significantly downregulated $(\mathrm{P}<0.001)$ by $>2$-fold in 46 pairs (78\%) of HCC tissues when compared with their normal counterparts (Fig. 2B). The reduced expression of KLF4-003 was found significantly associated with HCC recurrence (Table I, $\mathrm{P}=0.045$ ) in the follow-up of $31 \mathrm{HCC}$ patients. However, no correlation was detected between KLF4-003 expression level and other clinical parameters including sex, age, tumor size, differentiation status, The American Joint Committee on Cancer (AJCC) staging and HBV infection status (data not shown). 

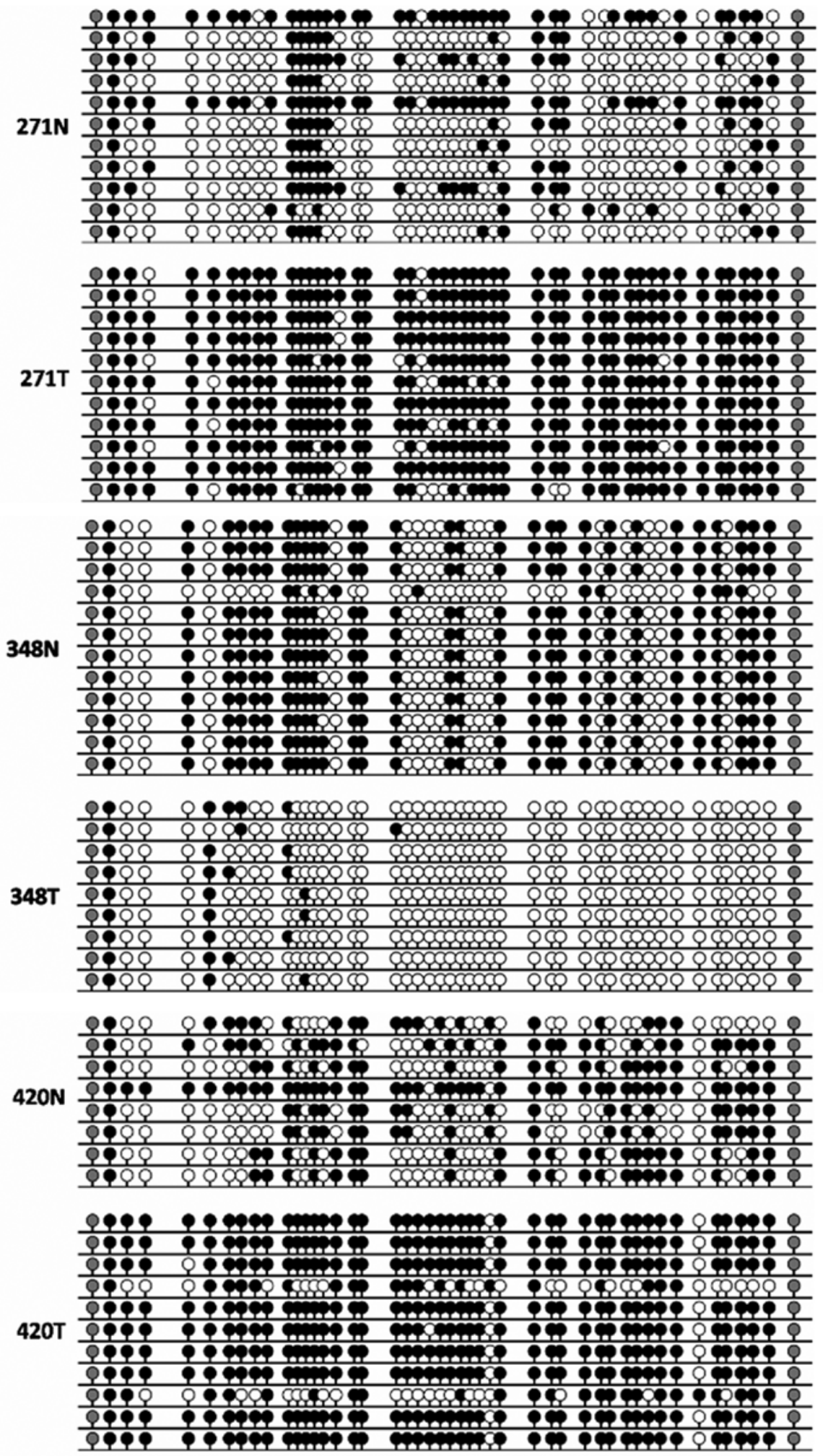

Figure 3. Methylation status of KLF4-003 CpG islands in liver cancer patient samples. Methylation status of CpG sites was examined by bisulfite sequencing. Open and filled circles represent unmethylated and methylated $\mathrm{CpG}$ sites, respectively.

Hypermethylation of non-promoter CpG island of KLF4-003 in $\mathrm{HCC}$ tissues. Epigenetic regulation has been closely related to altered gene expression. One feature of KLF4-003 is that it retains an intron of KLF4. Interestingly, a non-promoter $\mathrm{CpG}$ island proximal to this unique intron was observed. To investigate whether this $\mathrm{CpG}$ island is responsible for the decreased expression of KLF4-003 in HCC, we first determine the meth- ylation status of this $\mathrm{CpG}$ island in $\mathrm{HCC}$ samples by bisulfite sequencing. Significant differences of methylation status were detected between three pairs of investigated HCC specimens and their paracancerous tissues (Fig. 3).

Demethylation rescues the expression of KLF4-003 in Hep3B cells. To investigate whether the reduced expression of 

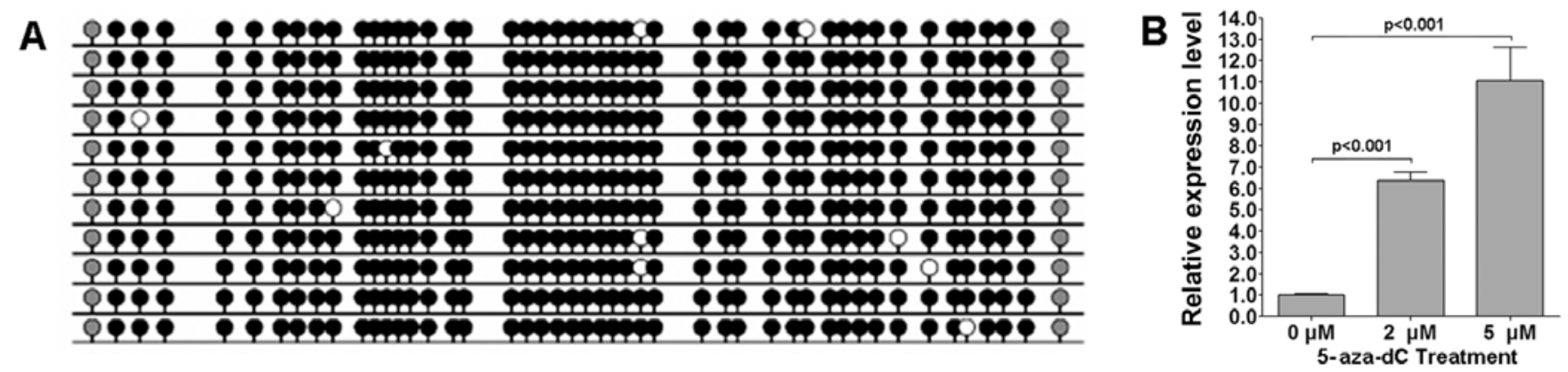

Figure 4. Expression of KLF4-003 rescued upon demethylation treatment in Hep3B cells. (A) Hypermethylation status of target CpG sites of KLF4-003 gene in Hep3B cells was determined by bisulfite sequencing. (B) The Hep3B cells were treated with different concentrations of 5-aza-dC for four days. Expression of KLF4-003 was significantly induced by both 2 and $5 \mu \mathrm{M}$ 5-aza-dC in Hep3B cells dose-dependently.
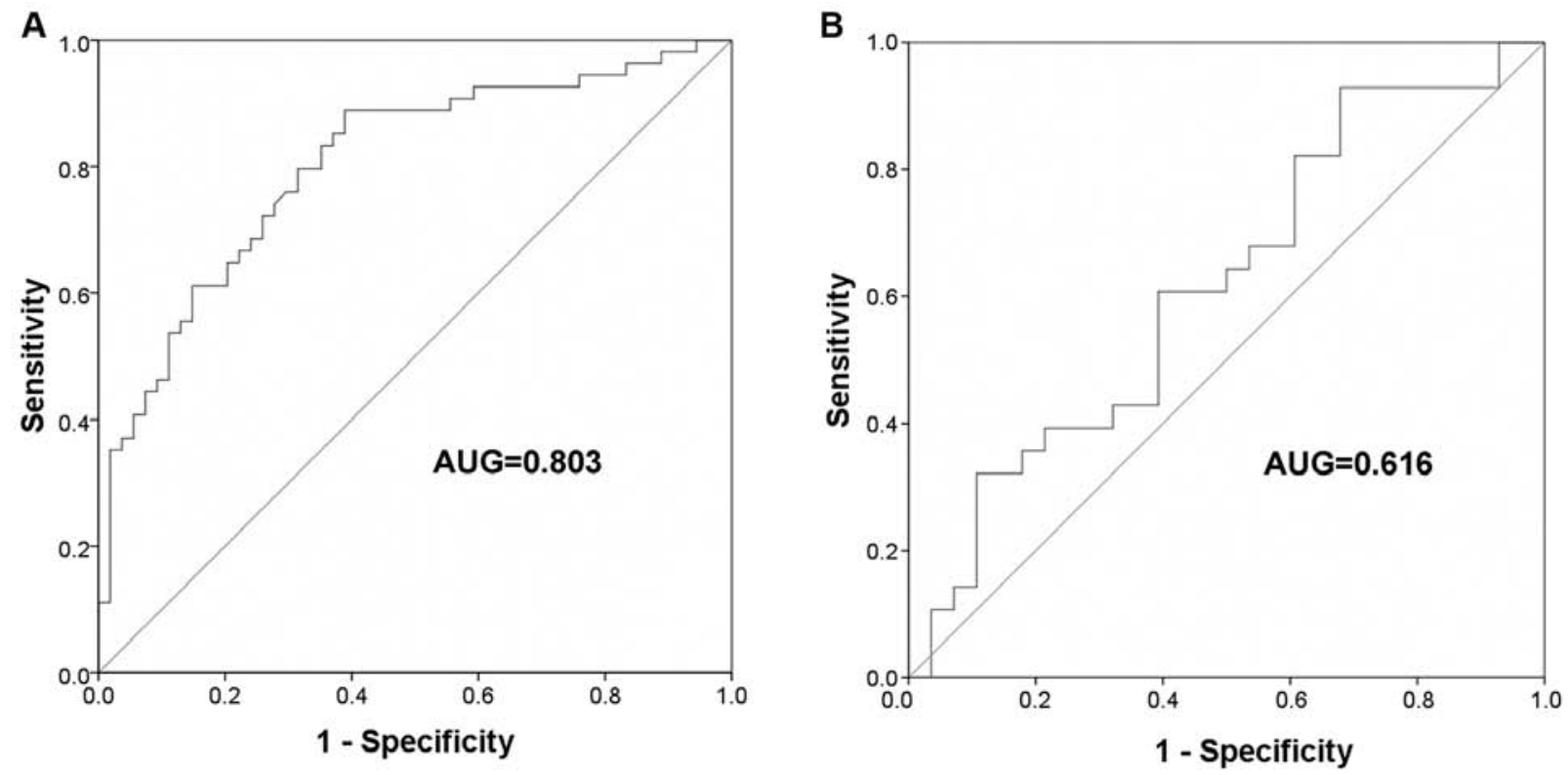

Figure 5. Receiver operation characteristics (ROC) curves. (A) ROC curve of using KLF4-003 as a marker, $\mathrm{P}<0.001$; (B) ROC curve of using KLF4 as a marker, $\mathrm{P}=0.136$.

KLF4-003 was caused by hypermethylation of the proximal non-promoter $\mathrm{CpG}$ island proximal to the retained intron, we determined the methylation status of this $\mathrm{CpG}$ island in Hep3B cells. As shown in Fig. 4A, hypermethylation was observed in the selected region. Then we treated the Hep3B cells with two concentrations of 5-aza-dC for 4 days. The expression of KLF4-003 was examined by RT-PCR. We found that KLF4-003 was significantly increased in Hep3B cells treated with 2 and $5 \mu \mathrm{M}$ of 5 -aza-dC in a dose-dependent manner compared with untreated cells (Fig. 4B). This finding suggested that hypermethylation of the selected non-promoter $\mathrm{CpG}$ island might be responsible for the reduced expression of KLF4-003 in HCC.

Observation of the value of using KLF4-003 as a diagnostic marker. To evaluate the diagnostic significance of KLF4-003 in HCC, the differences of KLF4-003 expression between $\mathrm{HCC}$ tissue and matched paracancerous tissues were compared based on the cutoff value (0.778) from the ROC curve. The area under ROC curve (AUC) reached 0.803 (95\% CI=0.719-0.886,
$\mathrm{P}<0.001$, Fig. 5A). The sensitivity was 0.889 and specificity was 0.389 . For comparison, ROC curve for KLF4 was also created (Fig. 5B). The AUC of KLF4 ROC curve was 0.616 $(95 \% \mathrm{CI}=0.468-0.764, \mathrm{P}=0.136)$. It was obvious that $\mathrm{KLF} 4-003$ had a better diagnostic value than KLF4.

KLF4-003 tends to remain in the nucleus. To investigate the cellular distribution of KLF4-003 RNA, RT-PCR was performed in WRL68 cells. In contrast to KLF4, the nuclear RNA level of KLF4-003 was >36-fold higher than the cytoplasmic RNA level (Fig. 6). Such observation was consistent with the property of the IncRNA.

KLF4-003 protein is endogenously undetectable in liver cells. Since KLF4-003 open reading frame (ORF) encodes a putative protein that is 34 amino acids longer than KLF4 protein, which enable KLF4-003 to be distinguished from KLF4 by western blot assay. To determine whether KLF4-003 was expressed in liver cells, the KLF4-003 ORF was cloned into pCMV-Myc vector and transiently transfected into Hep3B 


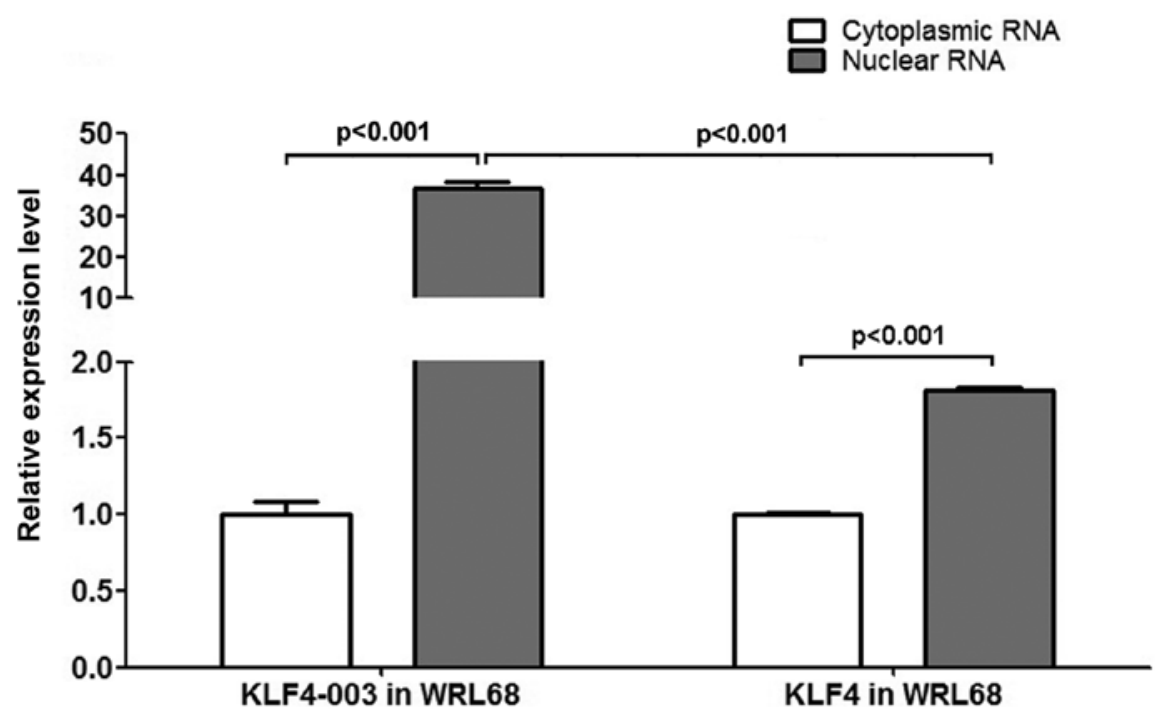

Figure 6. Differential cellular distribution of KLF4-003 in WRL68 cells. Nuclear RNA level of KLF4-003 was significantly higher than its cytoplasmic RNA level as well as the KLF4 nuclear RNA level.

A

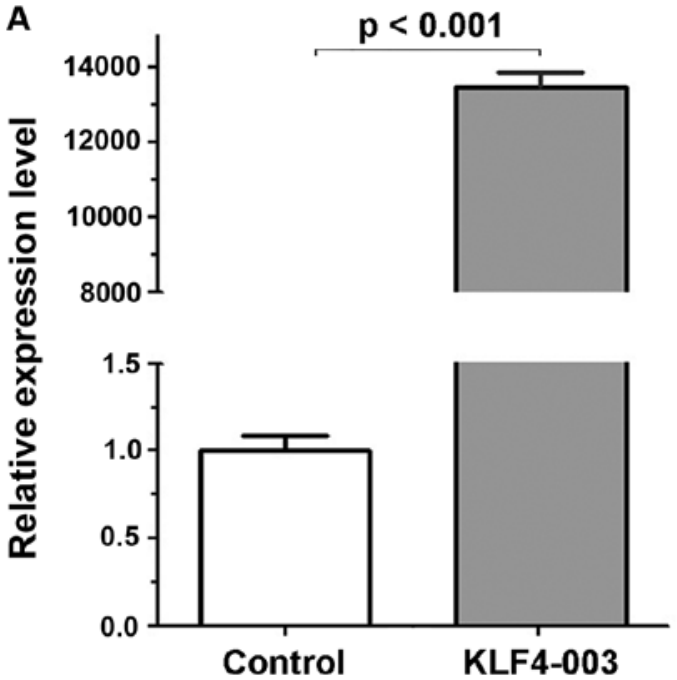

B

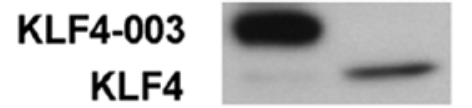

Anti-KLF4

$\beta$-actin
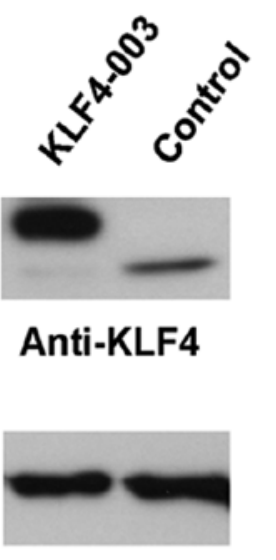

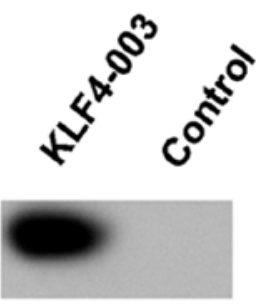

Anti-Myc

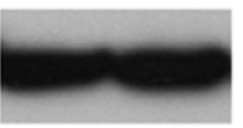

Figure 7. Endogenous KLF4-003 protein was undetectable in Hep3B cells. The KLF4-003 was transiently transfected into Hep3B cells with empty pCMV-Myc vector as control. (A) RT-PCR results indicated that KLF4-003 was highly overexpressed in Hep3B cells. (B) The KLF4-003 fusion protein with Myc tag and KLF4 was detected with anti-KLF4 antibody while the endogenous KLF4-003 was undetectable (left panel). The KLF4-003 fusion protein with Myc tag was also detected in Hep3B cells by using anti-Myc antibody (right panel). $\beta$-actin was used as control (the lower panel).

cells. We confirmed that the fusion protein KLF4-003 with Myc tag was detected, whereas, the endogenous KLF4-003 protein was undetectable (Fig. 7).

\section{Discussion}

Tumor markers have drawn increased attention as they could provide potential targets for diagnosis and therapeutic intervention. Recent studies have found the expression profiles of miRNAs were significantly altered in human neoplasms $(9,11)$, which could be used in tumor diagnosis to distinguish tumors from normal tissues. Despite the huge number of IncRNAs identified so far, definitely characterized lncRNAs only accounted for $<1 \%$ (19). Recent studies showed many lncRNAs were deregulated in various solid tumors and a number of IncRNAs could regulate cancer metastasis by directly targeting chromatin modification complexes, indicating that IncRNAs may play an important role in tumorigenesis and cancer development $(20,21)$. HCC is one of the most common malignant carcinomas in the world. It is of great importance to elucidate the functional role of lncRNA in HCC, which may significantly contribute to the better understanding, diagnosis and treatment of HCC conditions. An increasing number of evidence has suggested that deregulated expression of microRNAs (miRNAs) have considerable potential in predicting the prognosis of HCC patients $(22,23)$. In the present study, we identified a putative lncRNA, KLF4-003 in human HCC samples that clearly distinguishes HCC from their corre- 
sponding normal tissues. We demonstrated that HCC patients with lower KLF4-003 expression had a significantly increased risk of recurrence. ROC analysis indicated that the IncRNA KLF4-003 could serve as a potential tumor marker for HCC. Such observation further underlined the potential importance of the lncRNA in the molecular cell biology of neoplasia.

By comparative analyses, ncRNA promoters have been found more conserved than those of protein-coding genes (24). Many studies have linked the promoter-associated DNA methylation with transcriptional silencing of associated genes (25-28). Notably, non-promoter genomic DNA methylation has also been found within both intronic and exonic regions of numerous genes and could also elicit repressive effects on gene expression (29). By bisulfite sequencing, significant differences were detected between the methylation status of KLF4-003 in HCC specimens and their adjacent normal controls, which indicated that hypermethylation might be responsible for the downregulation of IncRNA KLF4-003. To verify the silencing effect of hypermethylation on KLF4-003, demethylation was performed in Hep3B cells. We found that the expression of KLF4-003 was dramatically rescued upon demethylation treatment in Hep3B cells. This observation supported the notion that non-promoter genomic DNA methylation could also result in gene silencing.

Much effort has been made to investigate the mechanisms of the relationship between DNA methylation and associated gene repression. At least four possible mechanisms have become apparent thus far. Firstly, the association of DNA-binding factors with their cognate DNA recognition sequences might be inhibited by the modification of cytosine bases (30). Secondly, methyl CpG binding proteins could recognize the methylated DNA, target chromatin remodeling co-repressor complexes and consequently mediate the silencing of gene expression (31). Thirdly, DNA methyltransferase enzymes themselves might be involved in setting up the silenced state besides their catalytic activities (32). Finally, transcriptional elongation could be affected by DNA methylation as the capacity of RNA polymerase II (Pol II) to transcribe through the methylated regions could be dampened by the DNA methylation (32). Although each of the above mechanisms may possibly elicit repressive effect on the expression of KLF4-003 in HCC, the exact role of this non-promoter $\mathrm{CpG}$ hypermethylation on KLF4-003 expression remains elusive.

It is interesting that KLF4-003 has an ORF although no endogenous protein product could be obtained. However, the majority of KLF4-003 was located in the nucleus. It has been shown that nuclear-localized lncRNAs are only transiently expressed and more likely unstable (33). The rapid degradation of nuclear-localized KLF4-003 may not permit sufficient KLF4-003 transcripts to complete the transportation from the nucleus to the cytoplasm for subsequent translation. It is notable that KLF4-003 fusion protein could be detected in Hep3B cells when highly overexpressed. Since the overexpressed KLF4-003 transcript did not contain 5'- and 3'-UTR, it is possible that the 5'- or 3'-UTR of endogenous KLF4-003 transcript contained protein binding sites which halted its transportation from the nucleus to the cytoplasm and thereby decreased the chance of this transcript to be translated in the cytoplasm. However, the exact mechanism for the predomi- nant nuclear localization of the KLF4-003 transcript remains unclear.

In conclusion, we identified the non-protein-coding transcript KLF4-003, which was downregulated in most of the examined HCC specimens compared with their paracancerous tissues. This is possibly due to the differential hypermethylation of the non-promoter CpG islands in the KLF4-003 gene. The reduced expression of KLF4-003 is significantly associated with the HCC recurrence and might serve as a potential diagnostic marker with high sensitivity for human HCCs.

\section{Acknowledgements}

The research team was supported by the Scheme B Funding on the Centre for Microbial Genomics and Proteomics from the Chinese University of Hong Kong, China.

\section{References}

1. El-Serag HB and Rudolph KL: Hepatocellular carcinoma: Epidemiology and molecular carcinogenesis. Gastroenterology 132: 2557-2576, 2007.

2. Blum HE: Hepatocellular carcinoma: Therapy and prevention. World J Gastroenterol 11: 7391-7400, 2005.

3. Soini Y, Chia SC, Bennett WP, Groopman JD, Wang JS, DeBenedetti VM, Cawley H, Welsh JA, Hansen C, Bergasa NV, et al: An aflatoxin-associated mutational hotspot at codon 249 in the p53 tumor suppressor gene occurs in hepatocellular carcinomas from Mexico. Carcinogenesis 17: 1007-1012, 1996.

4. Donato F, Tagger A, Gelatti U, Parrinello G, Boffetta P, Albertini A, Decarli A, Trevisi P, Ribero ML, Martelli C, et al: Alcohol and hepatocellular carcinoma: The effect of lifetime intake and hepatitis virus infections in men and women. Am J Epidemiol 155: 323-331, 2002.

5. Wei D, Wang L, Kanai M, Jia Z, Le X, Li Q, Wang H and Xie K: KLF4 $\alpha$ up-regulation promotes cell cycle progression and reduces survival time of patients with pancreatic cancer. Gastroenterology 139: 2135-2145, 2010.

6. Scherer SW, Cheung J, MacDonald JR, Osborne LR, Nakabayashi K, Herbrick JA, Carson AR, Parker-Katiraee L, Skaug J, Khaja R, et al: Human chromosome 7: DNA sequence and biology. Science 300: 767-772, 2003.

7. Ota T, Suzuki Y, Nishikawa T, Otsuki T, Sugiyama T, Irie R, Wakamatsu A, Hayashi K, Sato H, Nagai K, et al: Complete sequencing and characterization of 21,243 full-length human cDNAs. Nat Genet 36: 40-45, 2004.

8. Bartel DP: MicroRNAs: Genomics, biogenesis, mechanism, and function. Cell 116: 281-297, 2004.

9. Lu J, Getz G, Miska EA, Alvarez-Saavedra E, Lamb J, Peck D, Sweet-Cordero A, Ebert BL, Mak RH, Ferrando AA, et al: MicroRNA expression profiles classify human cancers. Nature 435: 834-838, 2005.

10. Meltzer PS: Cancer genomics: Small RNAs with big impacts. Nature 435: 745-746, 2005.

11. Volinia S, Calin GA, Liu CG, Ambs S, Cimmino A, Petrocca F, Visone R, Iorio M, Roldo C, Ferracin M, et al: A microRNA expression signature of human solid tumors defines cancer gene targets. Proc Natl Acad Sci USA 103: 2257-2261, 2006.

12. Erdmann VA, Barciszewska MZ, Szymanski M, Hochberg A, de Groot N and Barciszewski J: The non-coding RNAs as riboregulators. Nucleic Acids Res 29: 189-193, 2001.

13. Ji P, Diederichs S, Wang W, Böing S, Metzger R, Schneider PM, Tidow N, Brandt B, Buerger H, Bulk E, et al: MALAT-1, a novel noncoding RNA, and thymosin beta4 predict metastasis and survival in early-stage non-small cell lung cancer. Oncogene 22: 8031-8041, 2003.

14. O'Neill MJ: The influence of non-coding RNAs on allele-specific gene expression in mammals. Hum Mol Genet 14 (Suppl 1): R113-R120, 2005.

15. Steenman MJ, Rainier S, Dobry CJ, Grundy P, Horon IL and Feinberg AP: Loss of imprinting of IGF2 is linked to reduced expression and abnormal methylation of H19 in Wilms' tumour. Nat Genet 7: 433-439, 1994. 
16. Lottin S,Adriaenssens E, DupressoirT,Berteaux N, MontpellierC, Coll J, Dugimont T and Curgy JJ: Overexpression of an ectopic H19 gene enhances the tumorigenic properties of breast cancer cells. Carcinogenesis 23: 1885-1895, 2002.

17. Manoharan H, Babcock K, Willi J and Pitot HC: Biallelic expression of the H19 gene during spontaneous hepatocarcinogenesis in the albumin SV40 T antigen transgenic rat. Mol Carcinog 38: 40-47, 2003.

18. Hsu HT, Wu PR, Chen CJ, Hsu LS, Yeh CM, Hsing MT, Chiang YS, Lai MT and Yeh KT: High cytoplasmic expression of Krüppel-like factor 4 is an independent prognostic factor of better survival in hepatocellular carcinoma. Int J Mol Sci 15: 9894-9906, 2014.

19. Ponting CP, Oliver PL and Reik W: Evolution and functions of long noncoding RNAs. Cell 136: 629-641, 2009.

20. Calin GA, Liu CG, Ferracin M, Hyslop T, Spizzo R, Sevignani C, Fabbri M, Cimmino A, Lee EJ, Wojcik SE, et al: Ultraconserved regions encoding ncRNAs are altered in human leukemias and carcinomas. Cancer Cell 12: 215-229, 2007.

21. Tsai MC, Spitale RC and Chang HY: Long intergenic noncoding RNAs: New links in cancer progression. Cancer Res 71: 3-7, 2011.

22. Hou J, Lin L, Zhou W, Wang Z, Ding G, Dong Q, Qin L, Wu X, Zheng Y, Yang Y, et al: Identification of miRNomes in human liver and hepatocellular carcinoma reveals miR-199a/b-3p as therapeutic target for hepatocellular carcinoma. Cancer Cell 19: 232-243, 2011.

23. Ji J, Shi J, Budhu A, Yu Z, Forgues M, Roessler S, Ambs S, Chen Y, Meltzer PS, Croce CM, et al: MicroRNA expression, survival, and response to interferon in liver cancer. N Engl J Med 361: 1437-1447, 2009.

24. Carninci P, Kasukawa T, Katayama S, Gough J, Frith MC Maeda N, Oyama R, Ravasi T, Lenhard B, Wells C, et al; RIKEN Genome Exploration Research Group and Genome Science Group (Genome Network Project Core Group): The transcriptional landscape of the mammalian genome. Science 309: 1559-1563, 2005.
25. Boyes $\mathbf{J}$ and Bird A: DNA methylation inhibits transcription indirectly via a methyl-CpG binding protein. Cell 64: 1123-1134, 1991.

26. Compere SJ and Palmiter RD: DNA methylation controls the inducibility of the mouse metallothionein-I gene lymphoid cells. Cell 25: 233-240, 1981.

27. Kass SU, Landsberger N and Wolffe AP: DNA methylation directs a time-dependent repression of transcription initiation. Curr Biol 7: 157-165, 1997.

28. Siegfried Z, Eden S, Mendelsohn M, Feng X, Tsuberi BZ and Cedar H: DNA methylation represses transcription in vivo. Nat Genet 22: 203-206, 1999.

29. Hsieh CL: Stability of patch methylation and its impact in regions of transcriptional initiation and elongation. Mol Cell Biol 17: 5897-5904, 1997.

30. Watt F and Molloy PL: Cytosine methylation prevents binding to DNA of a HeLa cell transcription factor required for optimal expression of the adenovirus major late promoter. Genes Dev 2: 1136-1143, 1988.

31. Sarraf SA and Stancheva I: Methyl-CpG binding protein MBD1 couples histone $\mathrm{H} 3$ methylation at lysine 9 by SETDB1 to DNA replication and chromatin assembly. Mol Cell 15: 595-605, 2004.

32. Klose RJ and Bird AP: Genomic DNA methylation: The mark and its mediators. Trends Biochem Sci 31: 89-97, 2006.

33. Clark MB, Johnston RL, Inostroza-Ponta M, Fox AH, Fortini E, Moscato P, Dinger ME and Mattick JS: Genome-wide analysis of long noncoding RNA stability. Genome Res 22: 885-898, 2012. 